\title{
Manual of Fracture Management of Foot and Ankle (AO Trauma: Rammelt S, Swords M, Dhillon MS and Sands AK: Thieme Publishers)
}

Journal of Foot and Ankle Surgery (Asia Pacific) (2020): 10.5005/jp-journals-10040-1116

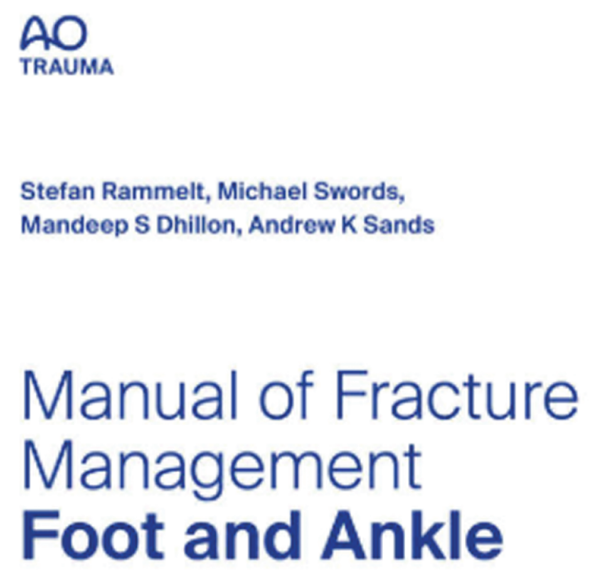

Thieme

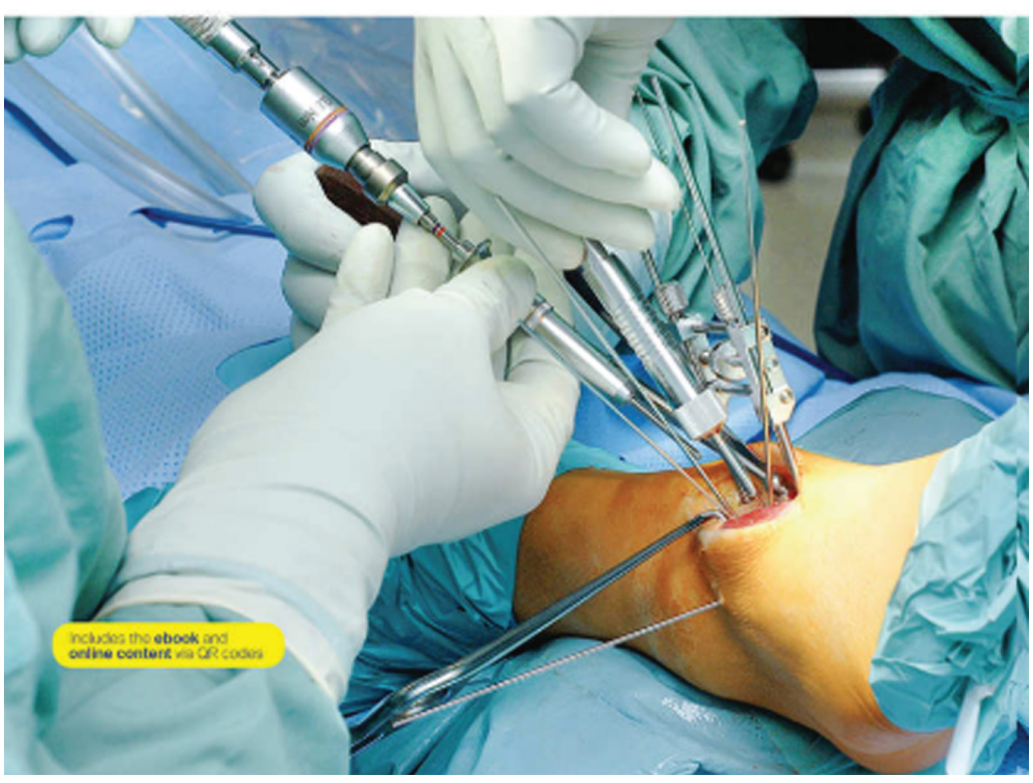

The Arbeitsgemeinschaft fur Osteosynthesefragen (AO) or the Association for the Study of Internal Fixation has become the premier innovator in the surgical treatment of bone fractures and disorders due to its revolutionizing outlook that goes from bench to bedside, including basic research, product development, clinical validation, and valorization. In 1969, Maurice Edmond Muller published the first AO manual on internal fixation in German which was translated in English by Schatzker next year. AO Education has strived to become more focused, by releasing a series of books specific to discrete areas of interest to surgeons. The AO manual on foot and ankle fracture management is the latest addition to this educational endeavor, with a prime focus on that part of the body whose alignment, congruity, and pain-free stability is paramount for locomotion. Unfortunately, the treatment of foot and ankle injuries is historically poorly understood, partly because of injuries of this appendage being considered minor to other injuries. One of the reasons for this could be limited proficiency in the current literature. This book is a major milestone in addressing this deficit.

(-) The Author(s). 2020 Open Access This article is distributed under the terms of the Creative Commons Attribution 4.0 International License (https://creativecommons. org/licenses/by-nc/4.0/), which permits unrestricted use, distribution, and non-commercial reproduction in any medium, provided you give appropriate credit to the original author(s) and the source, provide a link to the Creative Commons license, and indicate if changes were made. The Creative Commons Public Domain Dedication waiver (http://creativecommons.org/publicdomain/zero/1.0/) applies to the data made available in this article, unless otherwise stated. 
The Editors of this book hail from different parts of the world, i.e., 48 orthopedic surgeons from 14 countries which is a strong point of this tome for exchange of opinions regarding clinical protocols and scenarios of both developed and underdeveloped countries. The contributors are well versed with their specialties and have poured out their clinical experience in a unique and systematic casebased approach in various chapters of this book.

Beginning with the general considerations, this book is divided into sections discussing various parts of the foot and ankle. Each section is further subdivided into subsections, and the chapters progress with cases of increasing complexity. The unique selling point of this book is that each case is taken on as an individual chapter, with an elaborative discussion of all the aspects, spanning the diagnostic methods to the treatment choices, leading on to planning and ultimately the execution of the appropriate management. The surgical steps are lucidly outlined with vivid pictures and comprehensible diagrams and often with links to the vast online library (Fig. 1). An algorithmic approach to decision-making specific to the injury pattern makes this book highly resourceful, as each chapter replete with the management options of specific fractures becomes a stand-alone reading. Fifty-nine detailed cases covering a comprehensive range of foot and ankle injuries and more than 1,650 high-quality illustrations and images have been included. While a single case can be approached in a variety of ways, this book focuses on the important guidelines pertaining to most of the clinical scenarios faced when managing foot and ankle injuries.

The focus of this highly lucid book is both young surgeons and experienced ones, as there are details of applied anatomy of the foot and ankle, clinical and radiographic evaluation, preoperative planning, surgical approaches, and postoperative care and rehabilitation. This book is an essential read for every orthopedician aspiring to be a foot and ankle specialist and should be made available in the emergency operation rooms for all budding surgeons to refer in the time of need. To summarize, this practical, hands-on manual on the management of foot and ankle trauma is a "must have" for the orthopedic surgeons of any level.

Vishal Kumar Aman Hooda

Department of Orthopaedics, Postgraduate Institute of Medical Education and Research, Chandigarh, India, e-mail:amanhooda_10@yahoo.com 